\title{
Applied Neural Intelligence to Modeling, Control, and Management of Human Systems and Environments
}

\author{
Toly Chen, ${ }^{1}$ P. Balasubramaniam, ${ }^{2}$ Quek Hiok Chai, ${ }^{3}$ and Yi-Chi Wang ${ }^{1}$ \\ ${ }^{1}$ Department of Industrial Engineering and Systems Management, Feng Chia University, Taichung 407, Taiwan \\ ${ }^{2}$ Department of Mathematics, Gandhigram Rural University, Gandhigram 624302, India
}

${ }^{3}$ School of Computer Engineering, Nanyang Technological University, Singapore 639798

Correspondence should be addressed to Toly Chen, tcchen@fcu.edu.tw

Received 22 August 2012; Accepted 22 August 2012

Copyright (C) 2012 Toly Chen et al. This is an open access article distributed under the Creative Commons Attribution License, which permits unrestricted use, distribution, and reproduction in any medium, provided the original work is properly cited.

Planning, scheduling, and the control of resources and activities are key elements to survive and compete. Humans play a critical role in these activities. Actually, most systems could be viewed as an environment surrounded by human beings. Over the years, some novel solutions have been proposed to solve the problems of human systems and environments in modeling, control, and management. Advanced computing systems and artificial neural network approaches continue to be one of the most promising solutions.

The purpose of this special issue was to provide details on the development of advanced artificial neural network approaches and their applications to modeling, control, and the management of human systems and environments. The target audiences were researchers in information management, system engineering, environmental protection, as well as practicing managers and engineers. After a strict review, five articles from researchers around the world were finally accepted.

Predicting the stock market is an important facet of financial forecasting, attracting great interest from stock buyers and sellers, investors, policy makers, applied researchers, and many others who are involved in the capital market. Neural networks have been used extensively for stock market forecasting. S. Banik, M. Anwer, and M. K. Khan conducted a comparative study to predict the stock index values using soft computing models and a time series model. They used wellknown models such as the genetic algorithm (GA) model and the adaptive network fuzzy integrated system (ANFIS) model as soft computing forecasting models, while considering the generalized autoregressive conditional heteroscedastic $(\mathrm{GARCH})$ model as a time series model. The experimental results showed that the use of soft computing models is more successful than the time series model.

S. K. Boddhu and J. C. Gallagher described a novel frequency grouping based analysis technique, developed to qualitatively decompose the evolved controllers into explainable functional control blocks. They also provided a summary of their previous work related to evolving flight controllers for two categories of controllers and demonstrated the applicability of the newly developed decomposition analysis for both categories. Their proposed methodology has been successfully applied to autonomous and nonautonomous controllers, and it has been demonstrated that the methodology can indeed be used to decompose the evolved controllers into logically explainable control blocks for further control analysis.

K. H. Lim, K. P. Seng, and L.-M. Ang developed the Lyapunov theory-based radial basis function neural network (RBFNN) for traffic sign recognition. Their methodology, inserted multidimensional inputs into the RBF nodes, linked to multiple weights. An iterative weight adaptation scheme was then designed based on the Lyapunov-stability theory to obtain a set of optimum weights. After comparing the performances of the proposed classifier to some existing conventional techniques, the simulation results revealed that the proposed system achieved a better performance with a lower number of training iterations.

Drought forecasts can be an effective tool for mitigating some of the more adverse consequences of drought. A. M. Belayneh and J. F. Adamowski compared the effectiveness of three data driven models for forecasting drought conditions in the Awash River Basin of Ethiopia. The Standard 
Precipitation Index (SPI) was forecasted using an artificial neural network (ANN), support vector regression (SVR), and the wavelet neural network $(\mathrm{WN})$, and the performances were compared afterwards. The forecasting results indicated that the coupled wavelet neural network (WN) models were the best models for forecasting SPI values over multiple lead times in the Awash River Basin in Ethiopia.

T. Chen proposed a nonlinear programming and artificial neural network approach to optimize the performance of a job dispatching rule in a wafer fabrication factory. The proposed methodology fused two existing rules and constructed a nonlinear programming model for choosing the best values of the parameters in the two rules by dynamically maximizing the standard deviation of the slack, which has been shown in several studies to benefit the scheduling performance. In addition, a more effective approach was applied to estimate the remaining cycle time of a job, which was empirically shown to be conducive to improve the scheduling performance. Based on the experimental results, the optimization of the adjustable factors in the two rules is an appropriate tool for enhancing the scheduling performance of the dispatching rule.

\section{Acknowledgment}

We thank all the authors and reviewers for their valuable contributions to this special issue.

Toly Chen

P. Balasubramaniam

Quek Hiok Chai

Yi-Chi Wang 

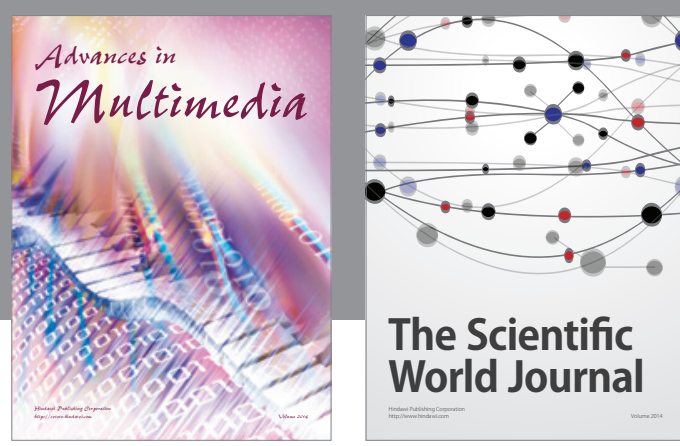

The Scientific World Journal
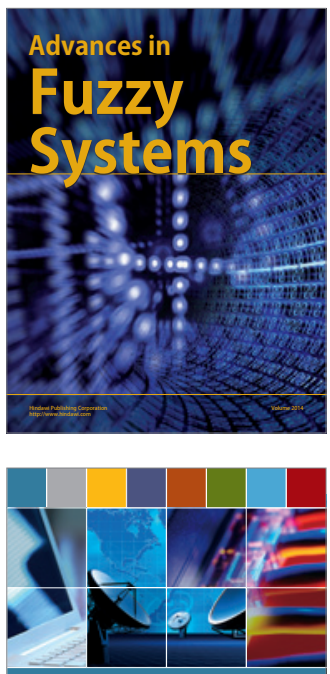

Computer Networks and Communications
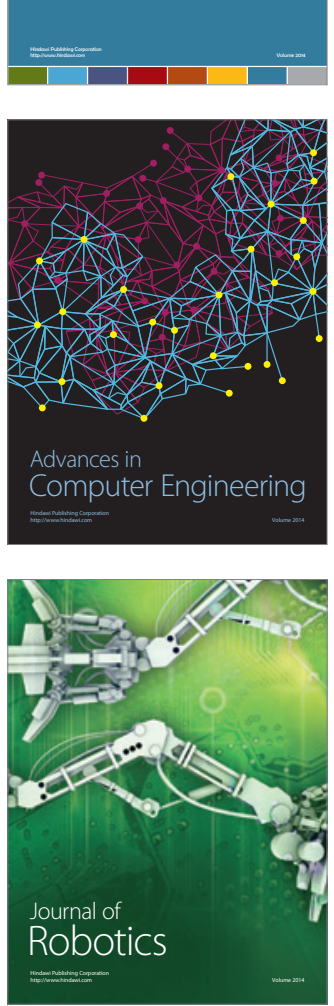
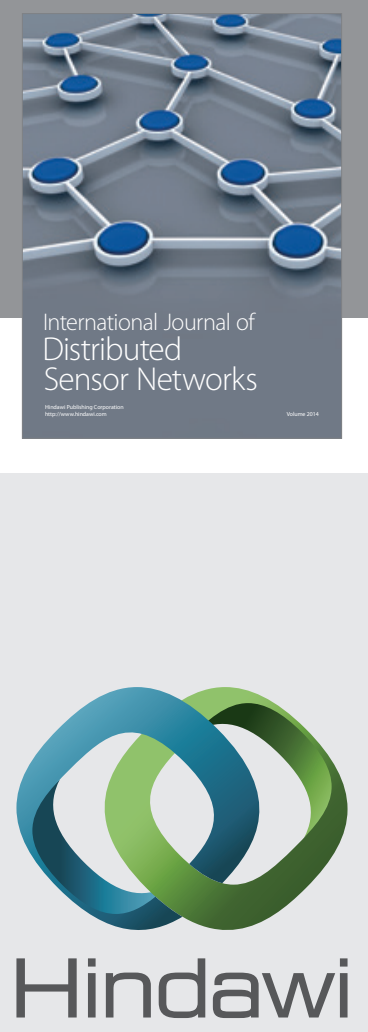

Submit your manuscripts at

http://www.hindawi.com
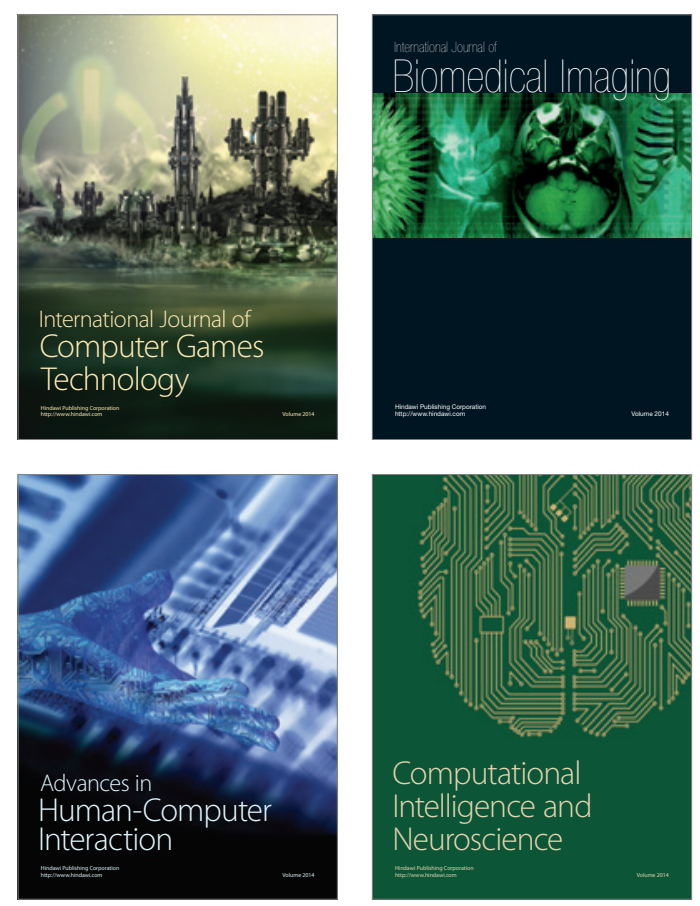
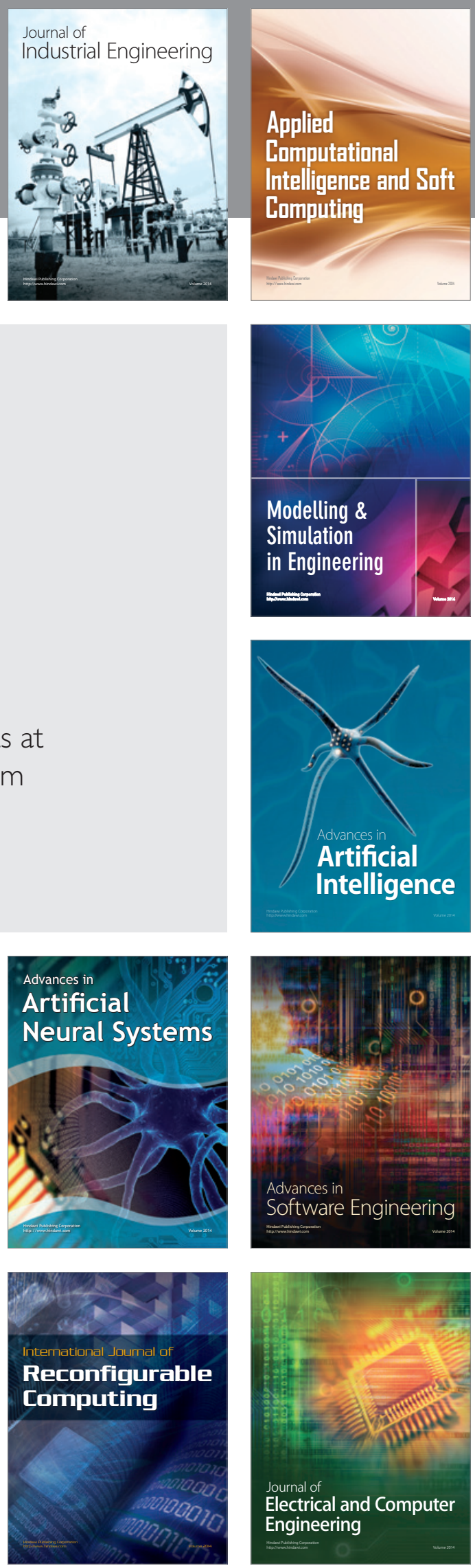